\title{
ISS outreach report
}

\author{
Rob Campbell • Mihra Taljanovic • Johnny Monu
}

Received: 27 November 2014 / Accepted: 4 December 2014 / Published online: 21 December 2014

(C) ISS 2014

The 2014 Sub-Saharan African ISS outreach program visited the Department of Radiology of Muhimbili University, Dar es Salaam in Tanzania. The United Republic of Tanzania is the largest country in East Africa and is the 13th largest country in all of Africa. The republic was formed in 1964 with the amalgamation of Tanganyika and the islands of Zanzibar, Peruba, and Mafia in the Indian Ocean. There are over 100 languages spoken by the indigenous population including Kiswahili, Arabic, Kiunguja, and English (Figs. 1, 2, and 3).

Tanzania is in the African Great Lakes region. It is bordered to the east by the Indian Ocean, to the west by the Republic of Congo, Zambia, Burundi, Rwanda, and Lake Tanganyika. The northern borders include Uganda, Kenya, and Lake Victoria. To the south, the neighbors include, Zambia, Malawi, and Mozambique. There is a population of 44.9 million, of which approximately $70 \%$ is rural in location. The official capital is the city of Dodoma. However, the port city of Dar es Salaam is the country's political capital, and principal commercial city, with a population of 4.6 million.

Tanzania is a relatively poor country on a par with other sub-Saharan countries. The economy is heavily dependent on agriculture, but with strong mining (gold and tanzanite gems) and construction sectors. In recent years, economic growth has been driven by tourism, telecommunications, and banking. The official poverty rate fell from $38.6 \%$ on 1991 to $33.4 \%$ in 2007 . Life expectancy is approximately 61 years. The under-five mortality rate is $54 / 1,000$ births. The leading

R. Campbell ( $\square)$

Department of Radiology, Royal Liverpool University Hospital,

Liverpool, UK

e-mail: rob.campbell@rlbuht.nhs.uk

M. Taljanovic

University of Arizona, Tucson, AZ, USA

J. Monu

University of Rochester, NY, USA causes of death in children are pneumonia, malaria, diarrhea, and prematurity. The prevalence of HIV/AIDS is $3.1 \%$.

The literacy rate is estimated at $73 \%$. Although education is compulsory for 7 years until the age of 15 years, it is estimated that only about $57 \%$ of children between the ages of 5 and 14 regularly attend school. Education up to the secondary school level is free, but those families who can afford it often educate their children in private schools, which are numerous. There are two state medical schools, one in Dar es Salaam and the other in Kilimanjaro. Medicine is a 6-year post-secondary school course and graduates are awarded Bachelor of Medicine and Bachelor of Surgery diplomas. After a 1-year internship, the young doctors have the option of practicing as general practitioners (non-specialists) or going into specialist training. Radiology is a 4-year graduate university course after which the trainees take the MSc degree exam. The graduating radiologists are awarded a Master of Science degree in Radiology.

The university campus incorporates the Muhimbili University of Health and Allied Sciences (MUHAS), the Muhimbili

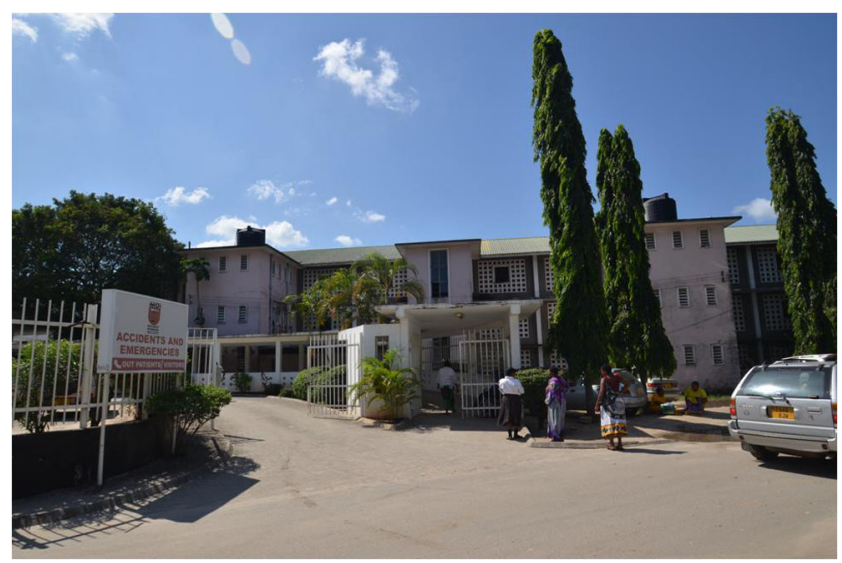

Fig. 1 Entrance to the Accident \& Emergency Dept at Muhimbili Orthopedic Institute 


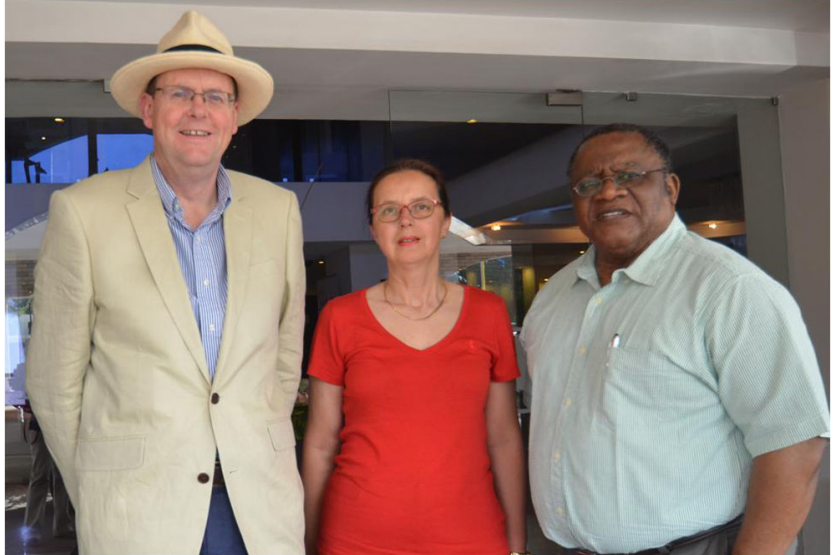

Fig. 2 ISS faculty: Dr. Rob Campbell, Prof. Mihra Taljanovic, and Prof. Johnny Monu

national hospital (MNH) and the Muhimbili Orthopedic Institute (MOI) and these facilities are also used as the teaching hospitals. Each of the institutions has an administratively independent radiology department. The MOI has limited imaging facilities at present with only radiography and US imaging available. However, more comprehensive imaging is available at $\mathrm{MNH}$, which includes four US rooms, one 1.5-T MR unit, and a 64-slice CT scanner. As of May 2014, construction for a new orthopedic institute was well advanced. This will incorporate a new radiology department, which will include both CT and MRI. There are other private hospitals scattered throughout Dar es Salaam that run their own independent, and usually, fully equipped radiology departments.

At MUHAS there are five radiologists who are lecturers. MNH has seven radiologists and one assistant radiologist and MOI has two radiologists. None of the radiologists are subspecialized although some have undergone short training electives lasting from a few months to a year in different subspecialties such as neuroradiology, breast imaging, pediatric radiology etc. outside Tanzania. The radiologists have trained in various countries including Cuba, Kenya, South Africa, and Uganda. Radiologists are currently trained at Muhimbili University in Dar es Salaam, and at Kilimanjaro University in Kilimanjaro. About four radiologists are trained every year in Muhimbili University.

The ISS outreach lecture program was held at MOI University, at the invitation of Dr. Lulu Fundikira, head of the

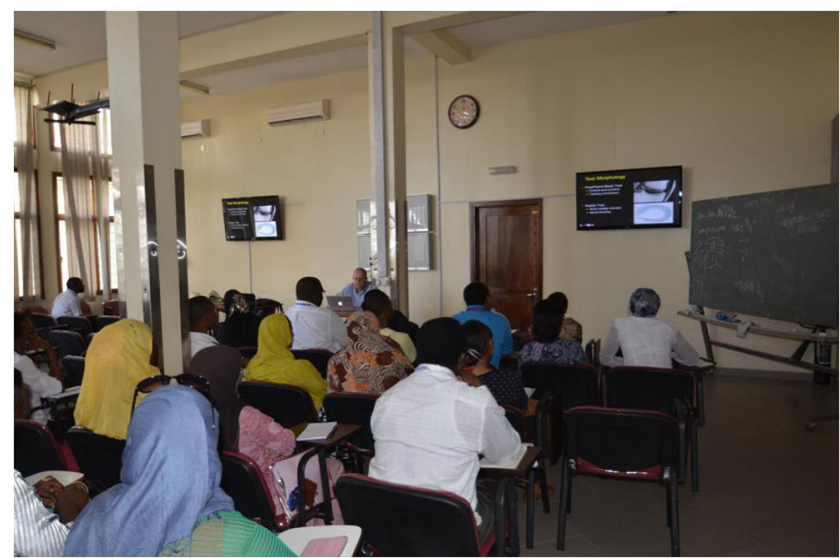

Fig 3 Teaching in progress in the Education Centre at Muhimbili Orthopedic Institute

Department of Radiology at Muhimbili University. The ISS faculty comprised Dr. Johnny Monu, Professor of Radiology from University of Rochester, NY, Dr. Mihra Taljanovic, Professor of Radiology from University of Arizona, Tucson, AZ, and Dr. Rob Campbell, Consultant Musculoskeletal Radiologist from Royal Liverpool University Hospital, UK. Our intrepid band of lecturers braved end-of-season monsoons, knee-deep potholes in the roads, legendary traffic congestion, and the biggest dengue fever outbreak in recent years.

A comprehensive program was run over 5 days, from May 12 to 16, 2014. The total of 20 lectures included a comprehensive discussion of internal joint derangements, by anatomical area, and included radiography, MRI, and US diagnosis. There were lectures on general topics including MSK infection, bone, and soft tissue tumors, diabetes, arthritis, and hemoglobinopathies. Professors Monu and Taljanovic provided interactive case reviews and Dr. Campbell gave US technique demonstrations within the radiology department.

The course proved very popular, with $20-30$ participants in attendance every day. The audience included university lecturers and radiology residents. All lectures were also provided in PDF format as a teaching resource for future residents. The popularity of the teaching program was reflected by the turnout at the end of program dinner, which was attended by the majority of the attending radiologists and the ISS faculty. 